\title{
Plasmaféresis terapéutica Experiencia del Hospital Universitario San Ignacio
}

\author{
Plasmaféresis terapéutiea \\ Experieneia delHospitalUniversitario San Ignaeio
}

\author{
Juan Pablo Córdoba, Carolina larrarte, Martín Alonso Rondón • \\ BogotÁ, D.C. (Colombia)
}

\section{Resumen}

Introducción: cada día hay más evidencia acerca de la utilidad de la plasmaféresis en diferentes entidades clínicas. Existen múltiples registros internacionales de plasmaféresis; sin embargo, la información en Colombia e incluso en latinoamérica es limitada.

Objetivo: realizar análisis descriptivo de la experiencia en plasmaféresis de una institución académica en Bogotá, Colombia. Analizar las características de la técnica, indicaciones, complicaciones y características demográficas de los pacientes.

Material y métodos: se realiza un análisis descriptivo de las sesiones de plasmaféresis realizadas en el Hospital Universitario San Ignacio, en pacientes mayores de 18 años durante el período comprendido desde agosto de 2008 hasta agosto de 2011. Todas las sesiones se realizaron mediante la técnica de filtración transmembrana y no se uso anticoagulante en ninguna de ellas.

Resultados: se analizaron 278 sesiones de plasmaféresis en 33 pacientes adultos durante un período de tres años. $69,7 \%$ de los pacientes eran mujeres, el promedio de edad era 42 años. 57\% de las sesiones se realizaron en pacientes con diagnóstico de miastenia gravis y hemorragia alveolar. El volumen promedio de intercambios plasmáticos fue 1,28. 9,3\% de las sesiones presentaron al menos una complicación. Se documentó frecuentemente hipotensión arterial en las sesiones realizadas con albúmina al 3,5\%. $12 \%$ de los registros de electrolitos tenían hipocalcemia y $47 \%$ hipermagnesemia. No hubo muertes relacionadas con la terapia y pese al no uso de anticoagulante, no se presentó coagulación del circuito en ninguna de las sesiones.

Conclusiones: dada nuestra experiencia, consideramos que la plasmaféresis es una terapia segura. Es necesario evaluar la necesidad de la administración de anticoagulante al circuito de plasmaféresis dado que no se documentó coagulación de éste en ninguna sesión y su uso podría estar asociado a eventos adversos. El monitoreo continuo de los electrolitos es fundamental y la reposición de los mismos debe ser individualizada. (Acta Med Colomb 2014; 39: 00-00).

Palabras clave: plasmaféresis, aféresis, intercambio plasmático terapéutico, anticoagulación.

\footnotetext{
Abstract

Introdtreeión: Cada día hay más evideneia aeerea de la utilidad de la plasmaféresis en diferentes entidades elínieas. Existen múltiples registros internaeionales de plasmaféresis; sin embargo, la información en Colombia e ineluso en latinoamériea es limitada.

Objetivo: Realizar análisis deseriptivo de la experieneia en plasmaféresis de una instittreión aeadémiea en Bogotá, Colombia. Analizar las earaeterístieas de la téeniea, indieaeiones, emmplieaeiones y earaeterístieas demográfieas de los paeientes.

Materiales y métodos. Se realiza un análisis deseriptivo de las sesiones de plasmaféresis realizadas en el Hospital Universitario San Ignaeio, en paeientes mayores de 18 años durante el perído eomprendido desde agosto de 2008 hasta agosto de 2011 . Todas las sesiones se realizaron mediante ta téeniea de filtraeión transmembrana y no se uso antieoagulante en nininguna de ellas.

Restltados: Se analizaron 278 sesiones de plasmaféresis en 33 paeientes adtultos durante un período de tres años. $69,7 \%$ de los paeientes eran mujeres, el promedio de edad era 42 años. $57 \%$ de las sesiones se realizaron en paeientes eon diagnóstieo de miastenia gravis y hemorragia alveolar. Et volumen promedio de intereambios plasmátieos fue 1,28. 9,3\% de las sesiones presentaron al menos una complieación. Se doeumentó freeuentemente hipotensión arterial en las sesiones realizadas con
}

Dr. Juan Pablo Córdoba Buriticá: Nefrólogo, Hospital Universitario San Ignacio. Jefe programa de Hemodiálisis y Aféresis, Profesor Pontificia Universidad Javeriana; Dra. Carolina Larrarte Arenas: Nefrólogo, Pontifica Universidad Javeriana. Médico Internista, Hospital Universitario San Ignacio; Dr. Martín Alonso Rondón Sepúlveda: Profesor de Bioestadística Universidad Javeriana. Bioestadístico, Departamento Epidemiología de la Pontificia Universidad Javeriana. Hospital universitario San Ignacio, Universidad Javeriana. Bogotá, D.C (Colombia). Correspondencia. Dr. Juan Pablo Córdoba Buriticá. Bogotá, D.C (Colombia).

E-mail: jpcordoba@husi.org.co

Recibido: 11/II/2013 Aceptado: 16/X/2013 
albúmina 3 l $3,5 \%$. $12 \%$ de los registros de eleetrolitos tenían hipoealeemia y $47 \%$ hipermagnesemia. No hubo mutrtes relaeionadas eon la terapia y pese al no uso de antieoagulante, no se presentó eoagulaeión del cireuito en ninguna de las sesiones.

Conelusiones: Dada nuestra experieneia, eonsideramos que la plasmaféresises una terapia segura. Es neeesario evaluar la neeesidad de la administraeión de antieoggutante al eiretrito de plasmaféresis dado que no se doeumentó coagulación de éste en ninguna sesión y su uso podría estar asociado a eventos adversos. El monitereo eontintu de los eleetrolitos es fundamental y la reposieión de los mismos debe ser individualizada. (Aeta Med Colomb 2014; 39: $\theta 0-\theta \theta$ ).

Key plasmapheresis, apheresis, therapeutic plasma exchange, anticoagulation.

\section{Introducción}

La plasmaféresis o intercambio plasmático terapéutico es un procedimiento de purificación sanguínea extracorpóreo, que se ha venido usando con mayor frecuencia en los últimos años y cada vez, con más indicaciones clínicas.

La primera plasmaféresis terapéutica se realizó en el año 1960 por Schwab y Fahey en un paciente con macroglobulinemia (1). Desde ese tiempo, han ido surgiendo diferentes indicaciones de este procedimiento, siendo inicialmente basadas en casos anecdóticos o estudios no controlados. Con el progreso del conocimiento médico y el entendimiento de la fisiopatología de varias enfermedades, el rol de la plasmaféresis se ha posicionado como parte del tratamiento de diferentes entidades clínicas. Actualmente, existe evidencia clínica que apoya el uso de este tipo de terapias en enfermedades renales, reumatológicas, hematológicas, neurológicas, infecciosas y metabólicas. Dentro de las indicaciones más frecuentes están la púrpura trombocitopénica trombótica, vasculitis ANCAS positivo con comportamiento rápidamente progresivo, miastenia gravis, Guilláin Barré, Síndrome de Goodpasture, entre otras (2-4).

Para la realización de la terapia se requieren equipos especializados que garanticen un tratamiento adecuado y seguro. Es necesario un sistema de circulación extracorpórea. La separación del plasma y las células sanguíneas se pueden realizar por métodos de centrifugación o mediante el uso de separadores de membranas, cada una de éstas teniendo ventajas y desventajas (5-7).

Existen registros internacionales de aféresis, la mayoría multicéntricos, realizados por servicios de hematología y basados en técnicas de centrifugación (8-12). Existen datos limitados en la población colombiana e incluso en la latinoamericana (13-17). Desde el año 2008, en el Hospital San Ignacio, el servicio de nefrología realiza plasmaféresis por técnica de filtración transmembrana. Se realiza un trabajo observacional descriptivo en el cual se describe la experiencia de la institución en esta terapia.

\section{Material y métodos}

Se realiza un análisis descriptivo de las sesiones de plasmaféresis realizadas en el Hospital Universitario San Ignacio, en pacientes mayores de 18 años durante el período comprendido desde agosto de 2008 hasta agosto de 2011. Se registran las características de la técnica, indicaciones, complicaciones y características demográficas de los pacientes, basado en información obtenida de la historia clínica de la institución y de la base de datos del grupo de aféresis del servicio de nefrología.

Se caracterizó la población por sexo, edad, raza, lugar de estancia hospitalaria, número de sesiones de plasmaféresis realizadas por paciente e indicación de la terapia.

Todas las sesiones de plasmaféresis se realizaron con un sistema de control volumétrico, sistema Aquarius (EdwardsBaxter), plasmafiltro plasma-flo Asahi y catéteres de hemodiálisis rectos y precurvados. No se administró anticoagulante en ninguna de las sesiones. El uso de anticoagulante, ya sea heparina o citrato, está descrito en la gran mayoría de textos y revisiones de aféresis como parte de la técnica; sin embargo, se consideró desde un inicio que su administración no es necesaria por protocolo, teniendo en cuenta que la plasmaféresis es un procedimiento corto, no existe evidencia contundente de que no usarlo afecte la eficacia de la terapia y su administración puede estar asociada a eventos adversos. Se administraron $2 \mathrm{~g}$ de gluconato de calcio y $2 \mathrm{~g}$ de sulfato de magnesio endovenoso durante todas las sesiones de plasmaféresis.

Se definió sesión como una terapia de plasmaféresis y procedimiento como el grupo de sesiones de plasmaféresis realizadas a un paciente, por un diagnóstico en particular y en un momento específico.

\section{Análisis estadístico}

Las variables categóricas se describen mediante medidas de frecuencia (proporciones y porcentajes) y las variables numéricas mediante medidas de tendencia central (promedio o mediana) y medidas de dispersión (desviación estándar).

\section{Resultados}

Se realizaron 278 sesiones de plasmaféresis correspondientes a 41 procedimientos, en 33 pacientes mayores de 18 años. $69.7 \%$ de los pacientes eran mujeres, el promedio de edad era 42 años, $6.1 \%$ de raza negra. Se intervinieron pacientes desde los 18 hasta 71 años y dos pacientes eran mayores de 65 años. El promedio de edad de las mujeres era mayor que el de los hombres. $54.6 \%$ de las sesiones de 
plasmaféresis fueron realizadas en Unidad de Cuidados Intensivos y el resto, en sala general y ambulatoria. El promedio de sesiones realizadas por paciente fue 8.4. Tabla 1 .

Las indicaciones de la terapia fueron 14 enfermedades correspondientes a enfermedades neurológicas, inmunológicas, reumatológicas, renales e intoxicaciones. El 57\% de los pacientes intervenidos cursaban con hemorragia alveolar y miastenia gravis. En el grupo de pacientes con hemorragia alveolar se encontraban pacientes con vasculitis de pequeño vaso ANCA positivo (seis pacientes), lupus eritematoso sistémico refractario (tres pacientes) y crioglobulinemia (un paciente). El promedio de sesiones por diagnóstico fue mayor en recurrencia de glomerulonefritis focal y segmentaria postrasplante renal y púrpura trombocitopénica trombótica. Tabla 2.

Todas las sesiones se realizaron por método de filtración transmembrana. En cuanto a los líquidos de reposición, se usó albúmina al 5\% en el 50.3\% de las sesiones, plasma en $28.1 \%$, albúmina al $3.5 \%$ en $19.8 \%$ y mezcla de plasma y albúmina en $1.8 \%$. La distribución del tipo líquido de resposición según diagnóstico se ilustra en la Figura 1. El plasma fue el líquido de reposición usado con mayor frecuencia en síndrome antifosfolípido catastrófico, púrpura trombocitopénica trombótica y hemorragia alveolar.

No se administró anticoagulante al circuito en ninguna de las sesiones de plasmaféresis. $80.4 \%$ de las sesiones se realizaron con catéteres transitorios, $14.1 \%$ con catéteres permanentes y $5.4 \%$ con fístulas arteriovenosas. $44.8 \%$ de los catéteres se localizaron en vena yugular y $55.2 \%$ en vena femoral.

Tabla 1. Características de los pacientes (33 pacientes - 278 sesiones de plasmaféresis).

\begin{tabular}{|l|c|}
\hline Característica & Valor \\
\hline Sexo - No (\%) & \\
Hombres & $10(30.3)$ \\
Mujeres & $23(69.7)$ \\
\hline Edad - años & \\
Media & $42.8 \pm 14$ \\
Rango & $18-71$ \\
\hline Edad según sexo - años & \\
Hombres & $38 \pm 7.7$ \\
Mujeres & $44.9 \pm 15.6$ \\
\hline Raza - No (\%) & $31(93.9)$ \\
No negra & $2(6.1)$ \\
Negra & \\
\hline Lugar estancia hospitalaria- & $1-51$ \\
No sesiones (\%) & $57(20.5)$ \\
Ambulatoria & $69(24.8)$ \\
Sala General & $152(54.6)$ \\
Unidad Cuidado Intensivo & \\
\hline Sesiones por paciente - No & \\
Promedio & \\
Rango & \\
\hline & \\
\hline
\end{tabular}

El promedio de número de recambios de volumen plasmático fue 1.28. El número de recambios de volumen plasmático realizados de acuerdo al diagnóstico se ilustra en la Tabla 3.

Tabla 2. Número de sesiones y pacientes según diagnóstico.

\begin{tabular}{|l|c|c|c|}
\hline $\begin{array}{l}\text { Diagnóstico } \\
\text { No(\%) }\end{array}$ & $\begin{array}{c}\text { Sesiones } \\
\text { No }\end{array}$ & $\begin{array}{c}\text { Pacientes } \\
\text { sesiones por } \\
\text { paciente }\end{array}$ & Promedio \\
\hline $\begin{array}{l}\text { Desensibilización pretrasplante } \\
\text { cardiaco* }\end{array}$ & $9(3,2)$ & 1 & 9 \\
Neuromielitis óptica & $5(1,8)$ & 1 & 5 \\
Guilläin Barré & $3(1,08)$ & 1 & 3 \\
Hemorragia alveolar & $54(19,4)$ & 10 & 5,4 \\
Intoxicación por cisplatino & $3(1,08)$ & 1 & 3 \\
Miastenia gravis & $77(27,6)$ & 9 & 8,5 \\
Neuropatía motora multifocal & $5(1,8)$ & 1 & 5 \\
Púrpura trombocitopénica trombótica & $30(10,8)$ & 2 & 15 \\
Rechazo humoral trasplante cardiaco* & $10(3,6)$ & 2 & 5 \\
Rechazo humoral trasplante renal & $16(5,7)$ & 2 & 8 \\
Recurrencia glomeruloesclerosis focal y & $51(18,3)$ & 1 & 51 \\
segmentaria postrasplante renal & & 1 & 5 \\
Síndrome de Goodpasture & $5(1,8)$ & 1 & 3 \\
Síndrome antifosfolípidos catastrófico & $7(2,5)$ & 1 & 5 \\
Encefalitis autoinmune & $3(1,08)$ & 1 & 5 \\
\hline * A una paciente se le realizan dos procedimientos de plasmaféresis indicados por dos \\
diferentes diagnósticos, desensibilización pretrasplante cardiaco y rechazo humoral \\
de trasplante cardiaco.
\end{tabular}

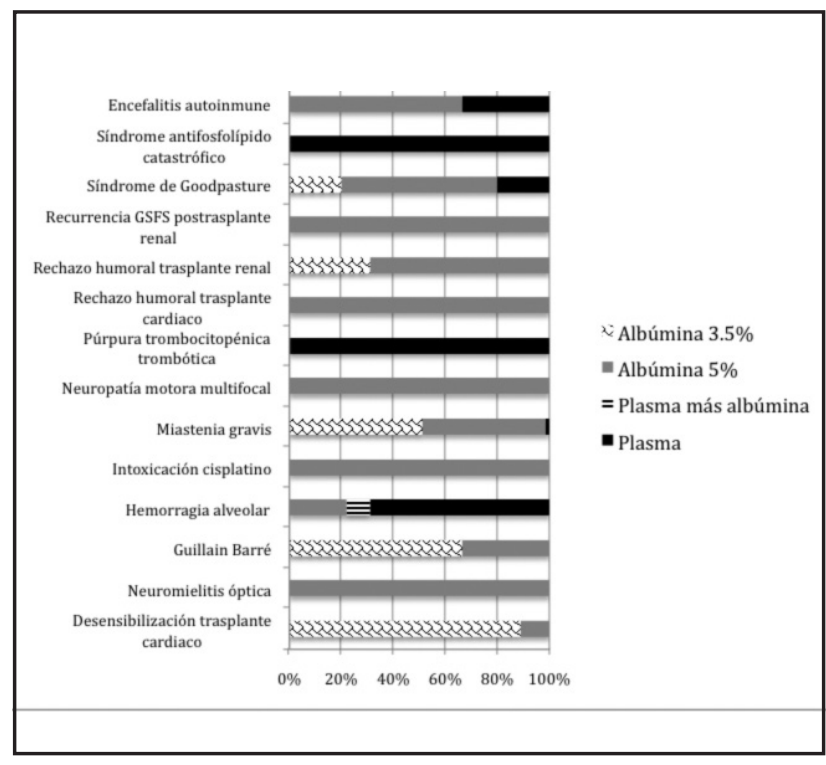

Figura 1. Distribución de líquido de reposición según diagnóstico. La albúmina al $5 \%$ fue usada frecuentemente como líquido de reposición. La distribución del líquido de reposición de acuerdo al diagnóstico muestra que en el síndrome antifosfolípido catastrófico, púrpura trombocitopénica trombótica y hemorragia alveolar, la solución usada con mayor frecuencia fue el plasma. FSGS: glomeruloesclerosis focal y segmentaria. 
Tabla 3. Número de recambios de volumen plasmático según diagnóstico.*

\begin{tabular}{|c|c|c|}
\hline $\begin{array}{l}\text { Diagnóstico } \\
\text { sesiones } \\
\text { analizadas }\end{array}$ & $\begin{array}{l}\text { Número de } \\
\text { de volumen } \\
\text { plasmático }\end{array}$ & Número de recambios \\
\hline Desensibilización pretrasplante cardiaco & 9 & $1.36 \pm 0.43$ \\
\hline Neuromielitis óptica & 5 & $0.96 \pm 0.21$ \\
\hline Guilläin Barré & 3 & $1.2 \pm 0.25$ \\
\hline Hemorragia alveolar & 54 & $1.28 \pm 0.22$ \\
\hline Intoxicación por cisplatino & 3 & $1.03 \pm 0.03$ \\
\hline Miastenia Gravis & 74 & $1.3 \pm 0.25$ \\
\hline Neuropatía motora multifocal & 5 & $1.2 \pm 0.02$ \\
\hline Púrpura trombocitopénica trombótica & 29 & $1.12 \pm 0.23$ \\
\hline Rechazo humoral trasplante cardiaco & 10 & $1.36 \pm 0.11$ \\
\hline $\begin{array}{l}\text { Rechazo humoral trasplante renal } \\
\text { Recurrencia glomeruloesclerosis focal }\end{array}$ & 16 & $1.28 \pm 0.29$ \\
\hline y segmentaria postrasplante renal & 41 & $1.36 \pm 0.24$ \\
\hline Síndrome de Goodpasture & 5 & $1.07 \pm 0.09$ \\
\hline Síndrome antifosfolípidos catastrófico & 7 & $1.4 \pm 0.1$ \\
\hline Encefalitis autoinmune & 3 & $1.45 \pm 0.11$ \\
\hline
\end{tabular}

En el $13 \%$ de las sesiones, los pacientes se encontraban con soporte vasoactivo o inotrópico y en $17 \%$, con ventilación mecánica.

Se presentaron complicaciones en $9.3 \%$ de las sesiones de plasmaféresis realizadas (39 complicaciones en 26 sesiones). El número máximo de complicaciones por sesión fue tres. Se presentó hipotensión arterial en 5.76\% de las sesiones, sólo uno de los 16 episodios de hipotensión requirió suspensión

Tabla 4. Complicaciones en 278 sesiones de plasmaféresis.

\begin{tabular}{|l|c|}
\hline Complicación & Porcentaje \\
\hline Hipotensión arterial & $5.76 \%$ \\
Asociadas a acceso vascular & $4.67 \%$ \\
Infección del acceso & $1.79 \%$ \\
Arritmias cardiacas & $0 \%$ \\
\hline
\end{tabular}

de la terapia. $4.67 \%$ de las sesiones presentaron complicaciones asociadas al acceso vascular, siendo la mayoría de estas, disfunción del acceso. Se registraron 4 episodios de sangrado del acceso vascular, uno considerado sangrado mayor dado que requirió drenaje de hematoma. Otras complicaciones derivadas del acceso vascular fueron punciones arteriales y fenómeno de robo. Se presentó infección del acceso vascular en $1.79 \%$ de las sesiones. No hubo registro de arritmias cardiacas asociadas a la terapia ni muertes derivadas de las complicaciones. No se presentó coagulación del circuito en ninguna sesión de plasmaféresis. Tabla 4.

Cuando se evalúa el porcentaje de sesiones complicadas según el líquido de reposición usado, se documenta que el $23.6 \%$ de las sesiones realizadas con albúmina al $3.5 \%$ presentó al menos una complicación. En orden descendente, se presentan complicaciones en $6.4 \%$ de las sesiones realizadas con plasma, $5.7 \%$ con albúmina al $5 \%$ y $0 \%$ con plasma más albúmina. En cuanto a hipotensión arterial, 10 de los 16 episodios registrados se presentaron en sesiones realizadas con albúmina al $3.5 \%$.

Los electrolitos se analizaron de forma independiente. Alrededor del $80 \%$ de los procedimientos de plasmaféresis tenían registro previo de magnesio, potasio y calcio dentro de límites normales. Un porcentaje significativo (67\%) tenían hiperfosfatemia, encontrándose con frecuencia en pacientes con disfunción renal concomitante. Las alteraciones electrolíticas documentadas antes del inicio de la plasmaféresis se registran en la Tabla 5.

Se analizan los controles de electrolitos y albúmina realizados a los pacientes durante el tiempo en que se encontraban en plasmaféresis y se documenta que el $85 \%$ de los registros de calcio se encontraban dentro de límites normales y $12 \%$ en rango de hipocalcemia, con una distribución similar del potasio. Se encontró un porcentaje significativo de hipoalbuminemia, $85 \%$. En cuanto al magnesio, el $47 \%$ de los registros se encontraban en rango de hipermagnesemia y $89 \%$ de estos, en rango de hipermagnesemia leve (magnesio entre 2.2 y $3.2 \mathrm{mg} / \mathrm{dL}$ ). No se registraron síntomas asociados a alteraciones electrolíticas. Figuras 2 y 3 .

\section{Discusión}

La plasmaféresis es una terapia que se viene usando en el mundo desde hace varios años, cada vez con mayor eviden-

Tabla 5. Control electrolítico antes del inicio de la plasmaféresis.**

\begin{tabular}{|c|c|c|c|c|c|}
\hline Electrolitos & Procedimientos evaluados - No $(\%)$ & Valor promedio $\pm \mathrm{DS}$ & Normal & Hipo & Hiper \\
\hline Magnesio & $20(49)$ & $1.95 \pm 0.21 \mathrm{mg} / \mathrm{dL}$ & $85 \%$ & $5 \%$ & $10 \%$ \\
\hline Potasio & $34(83)$ & $4.32 \pm 0.73 \mathrm{mEq} / \mathrm{L}$ & $82 \%$ & $6 \%$ & $12 \%$ \\
\hline Calcio & $18(44)$ & $9.21 \pm 0.79 \mathrm{mg} / \mathrm{dL}$ & $78 \%$ & $11 \%$ & $11 \%$ \\
\hline Fósforo & $15(37)$ & $4.99 \pm 1.2 \mathrm{mEq} / \mathrm{L}$ & $33 \%$ & $0 \%$ & $67 \%$ \\
\hline
\end{tabular}




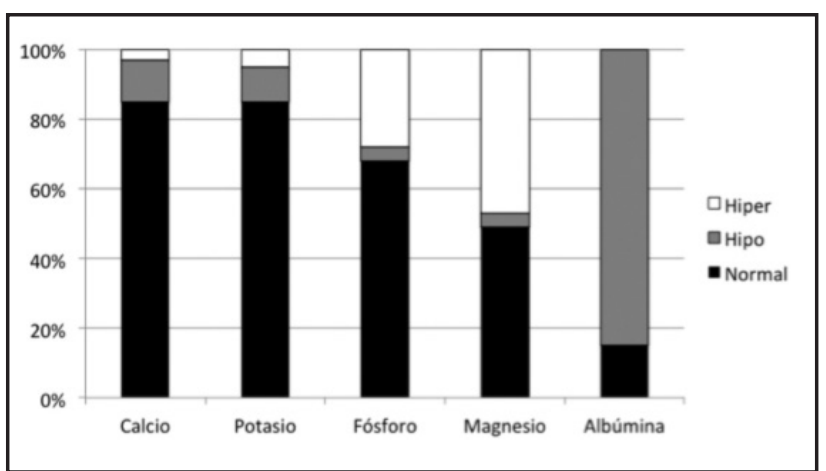

Figura 2. Control de electrolitos y albúmina. Esta gráfica muestra la distribución en categorías (normal, alto o bajo) de todos los registros de electrolitos realizados durante los procedimientos de plasmaféresis. Los registros de calcio y potasio tienen una distribución similar. $85 \%$ de los registros de calcio se encontraban en el rango de la normalidad, $12 \%$ en hipocalcemia. Los registros de hiperfosfatemia (28\%) se encontraron en pacientes con disfunción renal concomitante (datos no mostrados). $47 \%$ de los registros de magnesio se encontraban en rango superior al normal.

cia acerca de sus indicaciones y conocimiento sobre la técnica y complicaciones. Existen registros internacionales de aféresis; sin embargo, la información en Colombia e incluso en latinoamérica, es limitada. Hasta nuestro conocimiento, este registro se constituye en el más grande publicado en pacientes colombianos.

En el período estudiado se realizaron 278 sesiones en 33 pacientes adultos, todas las sesiones realizadas por técnica de filtración transmembrana. La mayoría de los registros de plasmaféresis están basados en terapias realizados por técnica de centrifugación a diferencia de las realizadas en nuestra institución.

Las indicaciones del procedimiento fueron 14 enfermedades correspondientes a enfermedades neurológicas, inmunológicas, reumatológicas, renales e intoxicaciones, la mayoría de ellas consideradas categoría I (enfermedades en las que la plasmaféresis se considera la primera opción de tratamiento) por la Sociedad Americana de Aféresis (ASFA, por sus siglas en inglés) (2). Un gran porcentaje de sesiones fueron realizadas a pacientes con miastenia gravis y hemorragia alveolar, diagnósticos reportados con frecuencia en la literatura. La mayor experiencia de la institución, en cuanto al número de sesiones de plasmaféresis, es en enfermedades neurológicas y renales. El promedio más alto de sesiones por paciente fue en recurrencia de glomeruloesclerosis focal y segmentaria en el injerto renal y púrpura trombocitopénica trombótica. Esto se explica porque en este tipo de enfermedades, la indicación de continuación de plasmaféresis depende de las respuesta clínica y paraclínica del paciente y no está sujeta a un número fijo de sesiones $(2,18,19)$.

Se realiza la mitad de las sesiones en Unidad de Cuidados Intensivos, reflejando la comorbilidad de los pacientes, y el grado de severidad de las enfermedades intervenidas. La dosis de la terapia en promedio fue de 1,28 recambios de volumen plasmático, acorde a lo indicado en la literatura (de 1 a 1,5 recambios de volúmenes plasmáticos) (2). En cuanto al líquido de reposición, inicialmente se usaba la albúmina

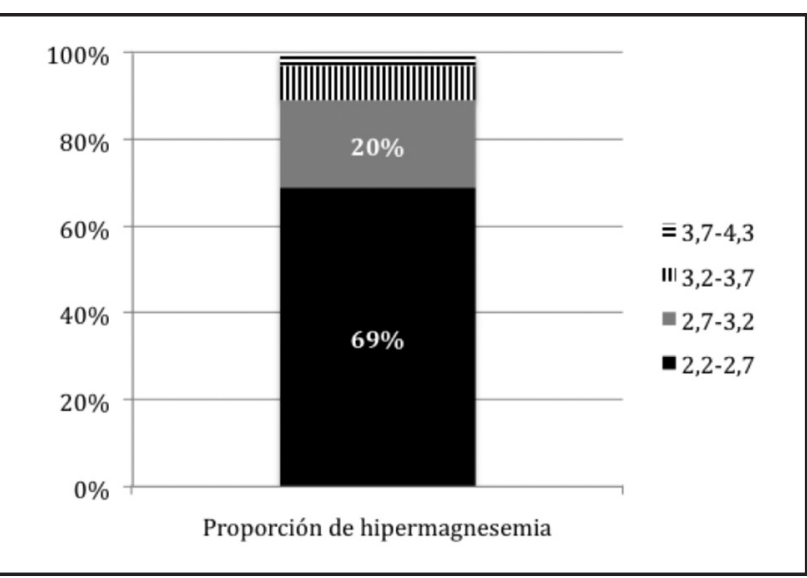

Figura 3. Distribución de hipermagnesemia. El rango de magnesio registrado fue de 1.1 a $4.3 \mathrm{mg} / \mathrm{dL}$. De los registros de hipermagnesemia, $89 \%$ se encontraban en rango leve $(2.2-3.2 \mathrm{mg} / \mathrm{dL})$.

al 3,5\% detectando que los pacientes presentaban con frecuencia hipotensión arterial durante las sesiones realizadas con este líquido. Por esta razón, el grupo de plasmaféresis decide realizar cambio de líquido de sustitución a albúmina al 5\%. Se usó plasma y mezcla de plasma más albúmina en pacientes con sangrado activo o riesgo de presentarlo y en pacientes con enfermedades que requerían la administración de plasma por el aporte de factores, como púrpura trombocitopénica trombótica $(5,20)$.

Alrededor del $10 \%$ de las sesiones presentaron al menos una complicación, que corresponde al porcentaje descrito en la mayoría de los registros de aféresis $(21,22)$. Sólo una complicación se consideró severa dado que requiere suspensión de la terapia (un episodio de hipotensión arterial) y no hubo mortalidad asociada a ninguna complicación. Cuando se analizan los episodios de hipotensión arterial, se detecta que la mayoría de estos ocurren en las sesiones realizadas con albúmina al $3.5 \%$ y que el porcentaje de sesiones complicadas realizadas con albúmina al $3.5 \%$ que presentan hipotensión arterial, es significativamente mayor que las complicadas realizadas con albúmina al $5 \%$ que presentan este mismo evento adverso.

No se presentó coagulación del circuito de plasmaféresis en ninguna sesión a pesar de que no se administró anticoagulante. Esto es un punto importante, teniendo en cuenta que la literatura recomienda la anticoagulación del circuito, ya sea con heparina o citrato, tanto en la técnica de filtración transmembrana como en centrifugación $(23,24)$.

Se documenta un porcentaje significativo de hipoalbuminemia probablemente asociado a la naturaleza inflamatoria de las enfermedades, a la cronicidad y comorbilidades de los pacientes. Las alteraciones electrolíticas se presentan con frecuencia durante el procedimiento de plasmaféresis. Se documenta que el $85 \%$ de los registros de calcio se encontraban dentro de límites normales y $12 \%$ en rango de hipocalcemia. En cuanto al magnesio, se documenta hipermagnesemia en $47 \%$ de los registros, hallazgo relacionado probablemente 
con la administración por protocolo de sulfato de magnesio. La mayoría de los registros de hipermagnesemia se encontraban en rango leve. No se registraron síntomas asociados a las alteraciones electrolíticas.

\section{Conclusiones}

Teniendo en cuenta la experiencia en plasmaféresis de la Unidad renal del Hospital Universitario San Ignacio, se considera que es una terapia segura que puede ser usada en diferentes escenarios clínicos. Es necesario evaluar la necesidad de administrar anticoagulante al circuito de plasmaféresis por técnica filtración transmembrana, dado que no se documentó coagulación del circuito en ninguna de las sesiones realizadas y su administración puede estar asociada a eventos adversos sistémicos como sangrado, hipocalcemia, entre otros. Esto debe ser evaluado cuidadosamente en futuras investigaciones teniendo en cuenta que los depósitos de fibrina en el plasmafiltro podrían afectar la eficacia de la terapia. En este registro de sesiones plasmaféresis, hubo respuesta clínica en la mayoría de los pacientes intervenidos, sugiriendo que la eficacia de la terapia probablemente no fue afectada (datos no mostrados).

Aunque la naturaleza descriptiva del estudio no permite establecer causalidad ni asociaciones, es frecuente encontrar hipotensión arterial en las sesiones realizadas con albúmina al $3.5 \%$ y en mayor porcentaje que en las realizadas con albúmina al 5\%, por lo que el grupo recomienda el uso de esta última en pacientes que no tengan indicación de plasma. Definitivamente, las alteraciones electrolíticas son frecuentes, por lo que debe realizarse monitorización continua de los electrolitos en los pacientes que son intervenidos con esta terapia. Se recomienda administrar gluconato de calcio y sulfato de magnesio según sean los niveles de calcio y magnesio de cada paciente, individualizando así la terapia.

\section{Agradecimientos}

María Nelcy Toro Quintero, jefe de enfermería de nefrología crítica, Hospital Universitario San Ignacio.

Andrea Sarmiento Agudelo, enfermera servicio de nefrología, Hospital Universitario San Ignacio.

\section{Declaración de fuentes de financiación y posibles conflictos de interés}

Este estudio no contó con ninguna fuente de financiación. Ninguno de los autores declaran conflictos de interés.

\section{Referencias}

1. Baweia S, Wiggins K, Lee D, Blair S, Fraenkel M, McMahon LP. Benefits and limitations of plasmapheresis in renal diseases: an evidence bases approach. J Artif Organs 2011; 14: 9-22.

2. Szczepiorkowski ZM, Winters JL, Bandarenko N, Kim HC, Linenberger ML, Marques MB, Sarode R, Schwartz J, Weinstein R, Shaz BH. Guidelines on the Use of Therapeutic Apheresis in Clinical Practice-Evidence-Based Approach from the Apheresis Applications Committee of the American Society for Apheresis. J Clin Apher 2011; 25: 83-177.

3. Kaplan AA. Therapeutic Plasma Exchange: Core curriculum 2008. Am J Kidney Dis 2008; 52: 1180-1196.

4. Moake JL. Thrombotic Microangiopathies. N Engl J Med 2001; 347: 589-600.

5. Ismail N, Kiprov DD, Hakim RM. Plasmapheresis. In: Daugirdas JT, Blake PG, Ing TS, editors. Handbook of dialysis, fourth edition. Lippincott Williams and Wilkins; 2007. p 275-298.

6. Floege J, Johnson RJ, Feehally J. Comprehensive Clinical Nephrology. 4th Edition, Elservier; 2010. p 1108-1118.

7. Sabath E, Denker BM. Plasmapheresis. In: Brenner BM, editor. Brenner and Rector's The Kidney. Philadelphia: Saunders Elsevier; 2008. p 2070-2079.

8. Korach JM, Guillevin L, Petitpas D, Berger P, Chillet P. Apheresis Registry in France: indications, techniques, and complications. Ther Apher 2000; 3: 207-210.

9. Stegmayr B, Ptak J, Wikstrom B. World apheresis registry report. Transfus Apher Sci 2007; 36: 13-16.

10. Bláha M, Pták J, Cáp J, Ceeová V, Masín V, Fillip S, Blazek M. WAA apheresis registry in the Czech Republic: two centers experience. Transfus Apher Sci 2009 41: 27- 31.

11. Yavasoglu I, Kadikoylu G, Akyol A, Bola Z. Therapeutic apheresis: Results from a single center in Turkey. Transfus Apher Sci 2007; 36: 249-253.

12. Stegmayr B, Ptak J, Wikström B, Berlin G, Axelsson CG, Griskevicius A, Centoni P, Liumbruno G, Molfettini P, Audzijoniene J, Mokvist K, Sojka BN, Norda R, Knutson F, Ramlow W, Blaha M, Witt V, Evergren M, Tomaz J World apheresis registry 2003-2007 data. Transfus Apher Sci 2008; 39: 247-254.

13. Restrepo CA, Márquez E, Sanz MF. Plasmaféresis terapéutica, tipos, técnica e indicaciones en medicina interna. Acta Med Colomb 2009; 34: 23-32.

14. Saltiel C. Apheresis Activity in Venezuela. J Clin Apher 2005; 20: 95-100.

15. Lazo-Langner A, Espinosa-Poblano I, Tirado-Cárdenas N, Ramírez-Arvizu P, López-Salmorán J, Peñaloza-Ramírez P, Sánchez-Guerrero SA. Therapeutic plasma exchange in Mexico: experience from a single institution. Am J Hematol 2002; 70: $16-21$

16. Benítez C, Andresen1 M, Farías G, Castillo C, Henríquez M, Pereira J. Uso de plasmaféresis en unidades de pacientes críticos. Rev Med Chile 2005; 133: 1441-1448.

17. Nogales-Gaete J, Valenzuela D, Liendo F, Vidal P, Gil G, Sáez D. Plasmaféresis indicada por enfermedad neurológica. Experiencia de 140 procedimientos en 47 pacientes. Rev Méd Chile 2004; 132: 295-298.

18. Keith DS. Therapeutic apheresis rescue mission: recurrent focal segmental glomerulosclerosis in renal allografts. Semin Dial 2012; 25: 190-192.

19. Clark WF. Thrombotic microangiopathy: current knowledge and outcomes with plasma exchange. Semin Dial 2012; 25: 214-219.

20. Ward DM. Conventional apheresis therapies: a review. Ward DMJ Clin Apher 2011; 26: 230-8

21. Kaplan A. Complications of Apheresis. Semin Dial 2012; 25: 152-158.

22. Mokrzycki MH, Balogun RA. Therapeutic apheresis: a review of complications and recommendations for prevention and management. J Clin Apher 2011; 26: 243-248.

23. Madore F. Plasmapheresis. Technical aspects and indications. Crit Care Clin 2002; 18: 375-392.

24. Lee G, Arepally GM. Anticoagulation techniques in apheresis: From heparin to citrate and beyond. J Clin Apher 2012; 27(3): 117-125. 